\title{
Quadro conceitual para gestão de estoques: enfoque nos itens
}

\section{A conceptual framework for inventory management: focusing on low consumption items}

\author{
Peter Wanke ${ }^{1}$
}

Resumo: Este artigo avalia a premissa da aderência da demanda à distribuição Normal em modelos de gestão de estoque, mostrando que tal consideração pode trazer distorções significativas no caso dos itens de baixo e baixíssimo consumo. Dessa forma, é proposto um quadro conceitual para auxiliar a identificação do modelo mais adequado com base nas principais características de cada item. Como contribuição adicional da pesquisa, é apresentada aplicação desse quadro conceitual no conjunto das peças de reposição de uma empresa brasileira, evidenciando-se seus principais benefícios em termos dos custos totais de falta e excesso.

Palavras-chave: Estoque. Demanda no tempo de resposta. Coeficiente de variação. Quadro conceitual. Custos.

\begin{abstract}
This article evaluates the premise of demand adherence to Normal distribution in inventory management models showing that such consideration can bring about significant distortions, mainly to very slow and slow moving items. Accordingly, the article proposes a framework to help identifying the best stock policy to be adopted, given the characteristics of each item. As an additional contribution, the paper presents an application of this framework on the set of spare parts of a Brazilian company making evident its main benefits in terms of shortage and excess costs.
\end{abstract}

Keywords: Stock. Lead time demand. Coefficient of variation. Framework. Costs.

\section{Introdução}

A gestão de estoque permeia a tomada de decisão em inúmeras empresas, sendo um tema bastante explorado no meio acadêmico e empresarial (ROSA; MAYERLE; GONÇALVES, 2010). As perguntas-chave que a gestão de estoque se propõe a responder, normalmente sujeitas a uma variedade de circunstâncias, são: quando pedir, quanto pedir e quanto manter em estoques de segurança (NAMIT; CHEN, 1999; SILVA, 2009). De acordo com Wanke (2011), a gestão de estoques abrange, portanto, um conjunto de decisões com o intuito de coordenar, nas dimensões tempo e espaço, a demanda existente com a oferta de produtos e materiais, de modo que sejam atingidos os objetivos de custo e de nível de serviços especificados, observando-se as características do produto, da operação e da demanda.

A maior preocupação das empresas no que tange a gestão de estoque pode ser atribuída, primeiramente, à necessidade de se garantir a maior disponibilidade de produto ao cliente final ao menor custo possível, dada a pressão competitiva dos mercados (EAVES, 2002). Além disso, alguns outros fatores contribuem para maior preocupação com a gestão de estoque: a diversidade crescente do número de produtos, que torna o comportamento ou padrão da demanda mais irregular (REGO; MESQUITA, 2011); e o elevado custo de oportunidade de capital, impactando os indicadores financeiros pelos quais as empresas são avaliadas (WANKE, 2011).

Segundo Silver, Peterson e Pyke (1998), Eaves e Kingsman (2004), Bai (2005), Syntetos, Boylan e Croston (2005) e Boylan, Syntetos e Karakostas (2008), o comportamento da demanda é um dos principais fatores que contribui para o aumento da complexidade dos modelos de gestão de estoque. Em linhas gerais, a demanda, pode ser classificada como: determinística ou randômica, de distribuição conhecida ou totalmente desconhecida e, ainda, ser independente e dependente (CORRÊA; CORRÊA, 2005; SILVA, 2009; WANKE, 2011).

O objetivo deste artigo é, portanto, explorar o padrão de demanda como principal fator interveniente na gestão de estoques. Inicialmente será discutido como as premissas frequentemente adotadas sobre a aderência da demanda à distribuição Normal podem não ser realistas e causar distorções, sobretudo

\footnotetext{
Centro de Estudos em Logística, Infraestrutura e Gestão - CELIG, Instituto COPPEAD de Administração, Universidade Federal do Rio de Janeiro - UFRJ, Cidade Universitária, Ilha do Fundão, CEP 21949-900, Rio de Janeiro, RJ, Brasil,

e-mail: peter@coppead.ufrj.br
} 
no caso dos itens de baixíssimo e baixo consumo (seção 2). Em seguida, na seção 3, será proposto um quadro conceitual para auxiliar na gestão de estoque, no qual são indicados os modelos mais adequados para um determinado padrão de demanda (média e variabilidade). Por fim, nas seções 4 e 5 , será apresentado um estudo de caso, conduzido em empresa brasileira, evidenciando-se não apenas a aplicação do quadro conceitual na prática, mas também o impacto, em termos de custos dos custos da falta e do excesso, oriundo dessa aplicação.

\section{Revisão de literatura}

A escolha do modelo de gestão de estoques mais adequado é, em essência, uma decisão de base empírica que pode envolver o uso de simulação, análises de cenários, análises de custos incrementais (SILVA, 2009; ROSA; MAYERLE; GONÇALVES, 2010; REGO; MESQUITA, 2011; WANKE, 2012) ou esquemas conceituais qualitativos, também conhecidos como abordagens de classificação (HUISKONEN, 2001). Esses últimos, normalmente, consideram o impacto das características do produto, da operação e da demanda como variáveis intervenientes nessa escolha (veja-se, por exemplo, WILLIAMS, 1984; HAX; CANDEA, 1984; DEKKER; KLEIJN; DE ROOIJ, 1998; BOTTER; FORTUIN, 2000; BRAGLIA; GRASSI; MONTANARI, 2004; EAVES; KINGSMAN, 2004; WANKE, 2012).

Pode-se afirmar também que a literatura disponível sobre seleção de modelos de gestão de estoque foi originalmente direcionada para ambientes de produção e distribuição nos quais a demanda e o tempo de resposta tendem a ser mais previsíveis, ou seja, nos quais as perguntas "quanto" e "quando pedir" são respondidas mais facilmente (WANKE; SALIBY, 2009; WANKE, 2010, 2012; ROSA; MAYERLE; GONÇALVES, 2010). Verifica-se, no entanto, o crescimento de literatura relacionada à problemática dos itens de baixo e baixíssimo consumo, como as peças de reposição (BOTTER; FORTUIN, 2000; SILVA, 2009; REGO; MESQUITA, 2011). Especificamente, as peças de reposição podem ser classificadas em duas categorias distintas que também impactam a seleção dos modelos de estoque mais adequados (SILVER; PETERSON; PYKE, 1998; BOTTER; FORTUIN, 2000). Os itens reparáveis são aqueles recuperáveis do ponto de vista técnico e econômico. Podem ser reparados em caso de falha, sendo disponibilizados novamente no estoque. Em contrapartida, os itens consumíveis são aqueles que, em caso de falha, são definitivamente descartados.

De todo modo, as características das peças de reposição, típicos itens de baixo e baixíssimo consumo, indicam por que a escolha de modelos de gestão de estoques é particularmente crítica nessas circunstâncias (COHEN; LEE, 1990; COHEN; ZHENG; AGRAWAL, 1997; MUCKSTADT, 2004; KUMAR, 2005; REGO, 2006): baixo giro dos estoques, difícil previsibilidade, tempo de ressuprimento mais elevado, maiores exigências de nível de serviço e maior custo de aquisição. Outro aspecto importante a ser ressaltado é o impacto causado pela ruptura desses itens que, na maioria das vezes, provoca a paralisação de equipamentos e/ou de processos relevantes para a empresa. Segundo Costa et al. (2005), a dificuldade para seleção dos modelos de gestão de estoque é, frequentemente, potencializada pela falta de entendimento dos custos da falta e do excesso relativos a esses itens.

Dessa forma, as particularidades mencionadas em relação às peças de reposição trazem implicações à seleção dos modelos de gestão de estoque. Segundo Botter e Fortuin (2000), há um consenso de que as peças de reposição não podem ser gerenciadas por meio de modelos tradicionais (veja-se, por exemplo, aqueles apresentados em ROSA; MAYERLE; GONÇALVES, 2010). Basicamente as peças de reposição não se adéquam às principais premissas inerentes a tais modelos, como por exemplo, a aderência da demanda às funções de densidade de probabilidade simétricas e contínuas (SILVA, 2009).

As próximas subseções aprofundam essa discussão, vinculando as características da demanda (média e variabilidade) aos modelos de gestão de estoque existentes na literatura. Especificamente com relação à demanda média, a literatura fornece as bases para a segmentação do consumo anual em três diferentes patamares: baixíssimo consumo, baixo consumo e consumo de massa. Já com relação à variabilidade, o coeficiente de variação e as funções de distribuição de probabilidade são as bases para segmentação.

\subsection{Baixíssimo consumo}

Segundo Tavares e Almeida (1983), as peças de baixíssimo consumo são consideradas aquelas que apresentam consumo médio inferior a uma unidade por ano. De acordo com esses autores, o controle de estoque desses itens não deve observar os modelos usualmente utilizados, uma vez que sua característica peculiar de consumo torna as ocorrências anteriores insuficientes para se estimar a distribuição de probabilidade com precisão (CROSTON, 1972; SYNTETOS; BOYLAN, 2001; GHOBBAR; FRIEND, 2003; EAVES; KINGSMAN, 2004; WILLEMAIN; SMART; SCHWARZ, 2004; REGATTIERI et al., 2005; HUA et al., 2007; GUTIERREZ; SOLIS; MUKHOPADHYAY, 2008; GOMEZ, 2008; TEUNTER; DUNCAN, 2009).

Ainda segundo Tavares e Almeida (1983), é a análise de custo total de falta, excesso e colocação de pedidos, dado um determinado nível de serviço, que permite determinar se deve ser mantida uma peça em estoque ou se é preferível não manter nenhuma peça, solicitando o ressuprimento apenas contrapedido. Dessa forma, um modelo binário de custo total foi desenvolvido para auxiliar a tomada 
de decisão com relação a manter sempre uma (1) ou nenhuma (0) unidade em estoque, com base em duas suposições: aderência da demanda à distribuição Poisson de parâmetro $\lambda$ e possibilidade de colocação de pedido emergencial, com tempo de resposta inferior ao usual, sempre que houver ruptura.

Partindo dessas suposições, os autores demonstraram que o custo total associado à política de não manter a peça de reposição em estoque (CT0) pode ser calculado da seguinte forma:

$$
C T 0=\lambda *(C T R+C i p)
$$

em que:

- $\lambda$ - consumo histórico médio (peças/ano);

- CTR - custo total de ressuprimento do item/ colocação do pedido (R\$); e

- Cip - custo de indisponibilidade e penalidade relativo à ruptura $(\mathrm{R} \$)$.

Para adotar a política alternativa, ou seja, a empresa mantém uma unidade em estoque até que o consumo ocorra, deve ser avaliada a fração de tempo esperada com estoque (FTECE), dada por:

$$
F T E C E=\frac{1}{1+\lambda * T R}
$$

em que:

- $T R$ - tempo de ressuprimento.

$\mathrm{O}$ valor esperado de ocorrências durante a fração de tempo esperada sem estoque é dada por $\lambda *(1-$ FTECE). Desta forma, pode-se obter o custo total associado à decisão de manter sempre uma peça em estoque (CT1), levando-se em consideração a possível ocorrência de outra solicitação durante o tempo de resposta, além de suas implicações em termos dos custos de ressuprimento e dos custos de indisponibilidade e penalidade, conforme é dado a seguir:

$$
\begin{aligned}
& C T 1=\left[\frac{1}{1+\lambda T R} * C a{ }^{*} i\right] \\
& +[C T R * \lambda]+\left[C i p * \lambda *\left(1-\frac{1}{1+\lambda * T R}\right)\right]^{(3)}
\end{aligned}
$$

As parcelas da Equação 3 representam, respectivamente, o custo de oportunidade de manter uma peça de reposição em estoque, o custo total de ressuprimento e o custo de indisponibilidade e penalidade. Para definir a política mais vantajosa, devem-se comparar as magnitudes de CT0 e CT1, optando-se pela decisão de menor custo.

\subsection{Baixo consumo}

Para efeito deste artigo, os itens de baixo consumo são aqueles que apresentam consumo histórico entre 1 e 300 unidades por ano, conforme sugerido por Wanke (2005). Uma vez que a demanda não é tão pequena para se utilizar o modelo proposto por
Tavares e Almeida (1983), as decisões relacionadas ao nível de serviço, tais como ponto de pedido e nível de reposição, assumem maior relevância. Dessa forma, o modelo (S,s), amplamente abordado na literatura, se torna mais adequado (ROSA; MAYERLE; GONÇALVES, 2010).

Mais precisamente, o modelo (S,s) envolve revisão contínua do nível de estoque, sendo os pedidos de ressuprimento feitos sempre que a posição do estoque atingir o ponto de pedido $s$ (SILVER; PETERSON, 1985; SILVER; PETERSON; PYKE, 1998). Neste caso, é utilizada uma quantidade de ressuprimento capaz de elevar a posição de estoque até o ponto $S$. Ou seja, na prática, o tamanho de lote é de (S). Segundo Hadley e Whitin (1961), os modelos (S,s) resultam em pedidos de tamanho de lote unitário quando os custos de colocação de pedido são baixos. Dessa forma, conforme conclusão de Feeney e Sherbrooke (1966), a política (S,S - 1) é um caso particular dos modelos $\mathrm{S}-\mathrm{s}$.

Diversos autores (FEENEY; SHERBROOKE, 1966; WALKER, 1997; PORRAS; DECKER, 2008; GOMES; WANKE, 2008) utilizaram o modelo $(\mathrm{S}, \mathrm{S}-1)$ na gestão de estoque de peças de reposição. Por meio deste modelo, assim que uma unidade de estoque é consumida, é solicitado um pedido de ressuprimento para recompor o nível máximo de estoque. Este modelo é adequado para componentes altamente dispendiosos e essenciais para a operação (WALKER, 1997).

O desempenho deste modelo é determinado exclusivamente pela decisão da quantidade a ser mantida em estoque $(S)$, como proteção contra faltas de estoques. Desta forma, caso o tempo de resposta seja instantâneo, $S$ deve ser igual a zero. Por outro lado, na presença de tempo de resposta não instantâneo, $S$ deve ser maior que zero (FEENEY; SHERBROOKE, 1966; PORRAS; DECKER, 2008; GOMES; WANKE, 2008).

Com relação às distribuições de probabilidade da demanda e do tempo de reposta no contexto dos modelos (S,s), Rosenshine et al. (1976, apud DHAKAR, SCHMIDT, MILLER, 1993) assumem inicialmente tempo de resposta determinístico. É importante ressaltar que, nesse caso, o tamanho do lote é diretamente proporcional à duração do tempo de resposta. Diversas distribuições, entretanto, já foram consideradas no contexto dos modelos $(\mathrm{S}, \mathrm{s})$ para representar o comportamento da demanda ou do tempo de resposta isoladamente: Normal (KRUPP, 1997), Gama (BURGIN, 1975; DAS, 1976; YEH, 1997), Poisson (HILL; OMAR; SMITH, 1999) e distribuição empírica com demandas e tempos de resposta estocásticos (EPPEN; MARTIN, 1988).

Outro ponto importante se refere à determinação da distribuição de probabilidade resultante pela combinação de resultados da demanda no tempo 
de resposta (LAU, 1989; SILVA, 2009). De acordo com Tyworth (1992), a distribuição Normal é uma aproximação razoável para itens de consumo elevado, mas não para itens de baixo consumo. Para estes itens, a distribuição é tipicamente assimétrica e possui alta probabilidade de demanda igual a zero. Porras e Decker (2008) adotaram a distribuição Poisson para estimar a demanda no tempo de resposta de itens com consumo unitário.

Cabe ressaltar que, quando o tamanho da demanda não é unitário, diversos autores têm proposto modelos compostos, como modelo de Poisson composto (WILLIAMS, 1984; SILVER; HO; DEEMER, 1971) ou modelo de Bernoulli composto (JANSSEN; HEUTS; KOK, 1998; STRIJBOSCH; HEUTS; SCHOOT, 2000). Entretanto, estes modelos são mais difíceis de serem aplicados na prática porque eles precisam da determinação dos parâmetros de mais de uma distribuição para representar o comportamento da demanda durante o tempo de resposta.

A escolha da distribuição mais adequada da demanda no tempo de resposta será abordada a seguir com maior profundidade.

\subsubsection{Distribuição Poisson}

Diversos modelos de gestão de estoque consideram a demanda no tempo de resposta aderente à distribuição Normal. Um aspecto subjacente a esta escolha é que os estoques apresentam padrão de demanda de alto consumo. Neste artigo, por exemplo, são considerados de alto consumo os itens que apresentam demanda histórica superior a 300 unidades por ano, conforme sugerido em Wanke (2005).

Já para itens de baixo consumo, Silver, Peterson e Pyke (1998) sugerem adotar a premissa da distribuição Poisson. Segundo Yeh (1997), entretanto, é necessário verificar inicialmente a aplicabilidade prática da distribuição Poisson. Isso porque, na distribuição Poisson, a média e a variância são numericamente iguais. Dessa forma, para que esta distribuição possa ser utilizada na prática, é necessário que a variância da demanda $(\operatorname{VAR}(D))$ esteja situada dentro de um intervalo delimitado por uma variação de $10 \%$ ao redor de sua média (E(D)), conforme sugerido pelo autor e indicado a seguir:

$$
0,9 * E(D)<V A R(D)<1,1 * E(D)
$$

Por ser discreta, a distribuição de Poisson fornece informações gerencialmente úteis, como, por exemplo, a probabilidade de ocorrência de um determinado nível de consumo, tomando-se como base sua média histórica. Além disso, pressupõe independência entre eventos, o que significa que o nível de consumo em um determinado período não é afetado pelo nível de consumo do período anterior. Por fim, na distribuição Poisson, o intervalo de tempo entre demandas segue uma distribuição exponencial (PORRAS; DECKER, 2008).

Especificamente, a distribuição Poisson é dada por:

$$
P_{x}(t)=\frac{(\lambda * t)^{x} e^{-\lambda t}}{x !}
$$

em que:

- $x$-consumo de peças de reposição por intervalo de tempo cuja probabilidade se deseja estimar;

- $t$-intervalo de tempo a ser considerado;

- $\lambda$ - taxa de consumo histórico por unidade de tempo; e

- $\mathrm{P}_{x}(t)$ - probabilidade de haver $x$ solicitações durante o intervalo de tempo $t$.

\subsubsection{Distribuição gama}

Conforme já abordado na seção anterior, a distribuição Normal não é recomendável nos casos de itens de baixo consumo, sendo a distribuição Gama uma alternativa à Poisson. A premissa da distribuição Gama foi adotada por Yeh (1997) em seu estudo, no qual mais de $50 \%$ dos itens considerados em sua amostra apresentavam consumo inferior a dez unidades por ano. A utilização de uma distribuição Poisson, originalmente foi descartada, pois os dados não satisfaziam a condição expressa na Equação 4. De acordo com Burgin (1975, apud YEH, 1997), a distribuição Gama é facilmente aderente aos dados reais, além de matematicamente manejável em aplicações de gestão de estoques.

De acordo com Yeh, Chang e Chang (1997), a distribuição Gama é adequada para os casos no quais os períodos com demandas nulas têm maior frequência de ocorrência. Nessas circunstâncias, faz sentido considerar o intervalo de tempo decorrido entre duas demandas consecutivas diferentes de zero como uma variável de interesse para a modelagem, além da própria demanda e do tempo de resposta. Segerstedt (1994, apud YEH, 1997), por exemplo, desenvolveu um modelo de gestão de estoques assumindo que o tempo entre duas demandas consecutivas não zero $\left(\mathrm{T}_{i}\right)$, a demanda $(\lambda)$ e o tempo de resposta $(T R)$ são aderentes à distribuição Gama.

A distribuição Gama é definida por dois parâmetros $\alpha$ e $\beta$, que são, respectivamente, o parâmetro de forma e o parâmetro de escala (KEATON, 1995). A função densidade de probabilidade da distribuição Gama é dada por:

$$
f(x)=\frac{(\alpha x)^{\beta-1}}{\Gamma(\beta)} \alpha e^{-\alpha x}, 0 \leq x<\infty
$$

em que:

- $\Gamma(\beta)=(\beta-1)$ !, quando $\beta$ é um inteiro; 
- $E(X)=\frac{\beta}{\alpha} ; \mathrm{e}$

- $\operatorname{Var}(X)=\frac{\beta}{\alpha^{2}}$.

O modelo utilizado por Yeh (1997) considerou como medida de nível de serviço a probabilidade de não haver falta em estoque durante um ciclo de pedido, ou seja, durante o intervalo de tempo entre dois ressuprimentos consecutivos. São definidos os níveis mais baixos e mais altos de nível de serviço desejados para cada item, de acordo com o modelo $(\mathrm{S}, \mathrm{s})$. Matematicamente, o nível de serviço para a posição de estoque remanescente é calculado por $1-\mathrm{P}_{\mathrm{S}}(\mathrm{S})$, no qual, $\mathrm{P}_{\mathrm{S}}(\mathrm{S})$ é a probabilidade de falta em estoque durante um ciclo de pedido, dado o nível de estoque $S$. Considerando-se que (S), $\mathrm{T}_{i}$ e $T R$ são aderentes à distribuição Gama, tem-se que:

$$
\begin{aligned}
& P S=\sum_{N=1}^{\infty} \frac{\gamma^{\delta}}{(\delta-1) !}\left\{\sum_{i=0}^{N \beta-1} \alpha^{i} \frac{(i+\delta-1) !}{i !(\gamma+\alpha)^{i+\delta}}\right. \\
& \left.-\sum_{i=0}^{(N-1) \beta-1} \alpha^{i} \frac{(i+\delta-1) !}{i !(\gamma+\alpha)^{i+\delta}}\right\} \\
& \times \mu^{N \sigma} e^{\mu \delta} \sum_{i=0}^{N \sigma-1} S^{i} \frac{1}{i ! \mu^{N \sigma-1}}
\end{aligned}
$$

em que:

- $(\mu, \sigma)$ - parâmetros da distribuição Gama acerca da demanda;

- $(\alpha, \beta)$ - parâmetros do intervalo de tempo entre duas demandas consecutivas não zero; e

- $(\gamma, \beta)$ - parâmetros do tempo de resposta de ressuprimento.

\subsection{Consumo de massa}

Frequentemente são considerados itens de consumo de massa aqueles que apresentam consumo histórico superior a 300 unidades por ano (WANKE, 2011). De acordo com Rosa, Mayerle e Gonçalves (2010), entre os principais modelos de gestão de estoque para itens de consumo de massa destaca-se o clássico $(P P, Q)$. De acordo com este modelo, $Q$ unidades são solicitadas sempre que a posição do estoque atingir o ponto de pedido PP (LOVE, 1979; SILVER; PETERSON; PYKE, 1998; MUCKSTADT, 2004; SHERBROOKE, 2004; HOPP; SPEARMAN, 2008). Na prática, o tamanho de lote $Q$ é determinado por meio da tradicional fórmula do Lote Econômico de Compras (HARRIS, 1913). Já o ponto de pedido é definido visando-se garantir uma determinada medida de nível de serviço (EPPEN; MARTIN, 1988; REGO et al., 2011).
Segundo Tyworth e Ganeshan (2000), de posse de informações sobre a demanda $(D)$ e os tempos de resposta $(T R)$ e partindo-se da premissa de que estas são variáveis aleatórias independentes, é possível determinar a média e a variância da demanda no tempo de resposta, partindo-se da média $(D)$ e da variância da demanda $(D)$, bem como da média $(T R)$ e da variância do tempo de resposta $(T R)$. As equações para se determinar a média e o desvio padrão da demanda no tempo de resposta são dadas a seguir, sendo uma discussão mais detalhada fornecida em Lau (1989):

$$
D_{T R}=D^{*} T R
$$

$$
S_{D^{*} T R}=\sqrt{\left(\operatorname{VAR}(D) * T R+\operatorname{VAR}(T R)^{*} D^{2}\right)}
$$

Num cenário sem incertezas, a taxa de consumo média dos produtos é previsível ao longo do tempo. Desta forma, é possível saber o momento de reabastecimento, ou seja, quando o nível de estoque chegará a zero (WANKE, 2011). Com isso, se a demanda e o tempo de resposta forem constantes, não há necessidade de formação de estoque de segurança (MENTZER; KRISHNAN, 1988). Neste contexto, o ponto de pedido $(P P)$, é o próprio valor da demanda no tempo de resposta $\left(D^{*} T R\right)$.

Por outro lado, conforme Speh e Wagenheim (1978), as incertezas da demanda e do tempo de resposta afetam diretamente a operação de um sistema de distribuição física. A incerteza da demanda está relacionada ao consumo que se materializa de maneira aleatória. Já a incerteza do tempo de resposta, por exemplo, decorre de atrasos ou antecipações. Para se proteger destes efeitos, torna-se necessária a formação de estoques de segurança $(E S)$.Com efeito, na presença de estoques de segurança, a fórmula do ponto de pedido passa a ser:

$$
P P=D * T R+E S
$$

em que:

$$
E S=K * S_{D^{*} T R}
$$

- D - demanda por unidade de tempo;

- $T R$ - tempo de resposta no ressuprimento, em unidades de tempo;

- $\mathrm{K}$ - fator de segurança, ou a quantidade de desvios padrão da demanda no tempo de resposta; e

- $\mathrm{S}_{\mathrm{D}^{* T R}}$ - desvio padrão da demanda no tempo de resposta.

É necessário conhecer o formato da distribuição da demanda no tempo de resposta para se determinar o estoque de segurança (KEATON, 1995). Conforme abordado por Porras e Decker (2008), esse cálculo requer que a distribuição da demanda durante o tempo de resposta seja especificada para que o valor do fator de segurança, $K$, seja determinado. Tradicionalmente, a demanda no tempo de resposta 
é modelada utilizando-se a distribuição Normal (SILVER; PETERSON, 1985). Pelas propriedades dessa distribuição, o fator de segurança $K$ para um determinado nível de serviço é igual aos valores tabelados na curva Normal padrão, $Z$, disponíveis em diversos livros de estatística e logística, como, por exemplo, Levine et al. (2005) e Ballou (2006).

Mentzer e Krishnan (1988) ressaltam que aproximar a demanda no tempo de resposta por uma distribuição Normal é diferente de considerar que a demanda e o tempo de resposta sejam normalmente distribuídos. Segundo estes autores, para qualquer distribuição da demanda e do tempo de resposta, os valores para a média e variância da demanda no tempo de resposta podem ser obtidos por meio das fórmulas (8) e (9), respectivamente. Para ser possível utilizar o valor de $Z$ no cálculo do ponto de pedido, a distribuição composta da demanda durante o tempo de resposta precisa aderir à Normal ou, pelo menos, que esta seja uma boa premissa.

No entanto, diversos trabalhos criticam essa aproximação. De acordo com Mentzer e Krishnan (1988), esta aproximação só é válida se a distribuição Normal for definida entre o intervalo de $-\infty$ a $+\infty$. Além disso, em muitas aplicações práticas, isto cria a possibilidade de demanda negativa. Por outro lado, para Moors e Strijbosch (2002), uma grande restrição da distribuição Normal é a premissa de simetria. Ademais, segundo Eppen e Martin (1988), somente em alguns casos é possível encontrar itens que apresentam uma distribuição Normal da demanda durante o tempo de resposta. Além disso, sua aproximação para determinar o ponto de pedido e estoque de segurança incorre em erros, levando a rupturas ou excesso de estoque. Por outro lado, Tyworth e O'Neill (1997) estudaram os efeitos provocados pela utilização da distribuição Normal em produtos de consumo de massa e concluíram que, apesar do fato de a aproximação pela distribuição Normal acarretar em imprecisões no dimensionamento do estoque de segurança, os erros na avaliação dos custos totais tendem a ser reduzidos.

Neste contexto, Silver, Peterson e Pyke (1998) propõem uma regra geral para a aproximação da demanda no tempo de resposta por uma distribuição de probabilidade de acordo com coeficiente de variação $(C V)$, no caso específico de itens de consumo de massa. Se $C V$ for maior do que 0,5, a distribuição Gama deve ser utilizada. Caso contrário, a distribuição Normal é uma boa aproximação para a demanda no tempo de resposta.

\section{Quadro conceitual proposto para gestão de estoques}

As diversas particularidades que devem ser observadas para adequada seleção dos modelos de gestão de estoque têm contribuído para o desenvolvimento de pesquisas e artigos sobre possíveis esquemas conceituais qualitativos, também conhecidos como abordagens de classificação, visando o apoio à tomada de decisão (HUISKONEN, 2001). O mais tradicional desses esquemas é a classificação $\mathrm{ABC}$, segundo a qual os itens são classificados de acordo com sua demanda, seu custo de aquisição e, consequentemente, sua representatividade financeira para o negócio. Um item A, por exemplo, pertence aos $20 \%$ que respondem por $80 \%$ do total de vendas da empresa (SILVER; PETERSON; PYKE, 1998).

Nas últimas décadas, merecem destaque alguns quadros conceituais. Williams (1984) desenvolveu um método analítico para classificar demanda como regular (alto consumo), baixo consumo ou intermitente pela decomposição da variância da demanda durante o tempo de resposta em três partes: variabilidade do número de ocorrências por unidade de tempo, variabilidade do tamanho da demanda e variabilidade do tempo de resposta. Botter e Fortuin (2000) classificam os itens a partir de três critérios, quais sejam: tempo de resposta, preço e nível de consumo, a partir dos quais são estabelecidos oito diferentes modelos de gestão de estoque. Já Eaves e Kingsman (2004) retomam o modelo de Williams (1984), reclassificando as peças de reposição em cinco categorias: suave, irregular, baixo giro, esporádica leve e esporádica forte. Por sua vez, Syntetos, Boylan e Croston (2005) classificam os itens em quatro quadrantes, considerando dois eixos: intervalo médio entre demandas e o quadrado do coeficiente de variação da demanda. Anos mais tarde, Boylan, Syntetos e Karakostas (2008) apresentam uma aplicação desse método em empresa de software. O padrão de consumo dos itens é classificado como: esporádico forte; esporádico leve; e não esporádico.

Tomando como ponto de partida o referencial teórico apresentado nas seções anteriores, é proposto um quadro conceitual para gestão de estoque com base na segmentação da demanda anual em três categorias - baixíssimo consumo, baixo consumo e consumo de massa - e do coeficiente de variação da demanda em duas - alta incerteza e baixa incerteza. Com base nessas duas variáveis associadas ao padrão da demanda, o quadro conceitual aponta qual o modelo de gestão de estoque mais adequado, apoiando a tomada de decisão à luz das premissas mais aderentes para responder às questões de quanto pedir, quando pedir e quanto manter em estoques de segurança.

No quadro conceitual, apresentado na Figura 1, são considerados como de baixíssimo consumo os itens com demanda média histórica inferior a uma unidade por ano. Os itens de baixo consumo correspondem aos itens cujo consumo médio histórico pode variar entre uma e $300 \sim 500$ unidades por ano, ou seja, no máximo uma unidade por dia. Em contrapartida, os itens considerados como consumo de massa são aquelas que apresentam demanda superior a aproximadamente $300 \sim 500$ unidades por ano. Já 


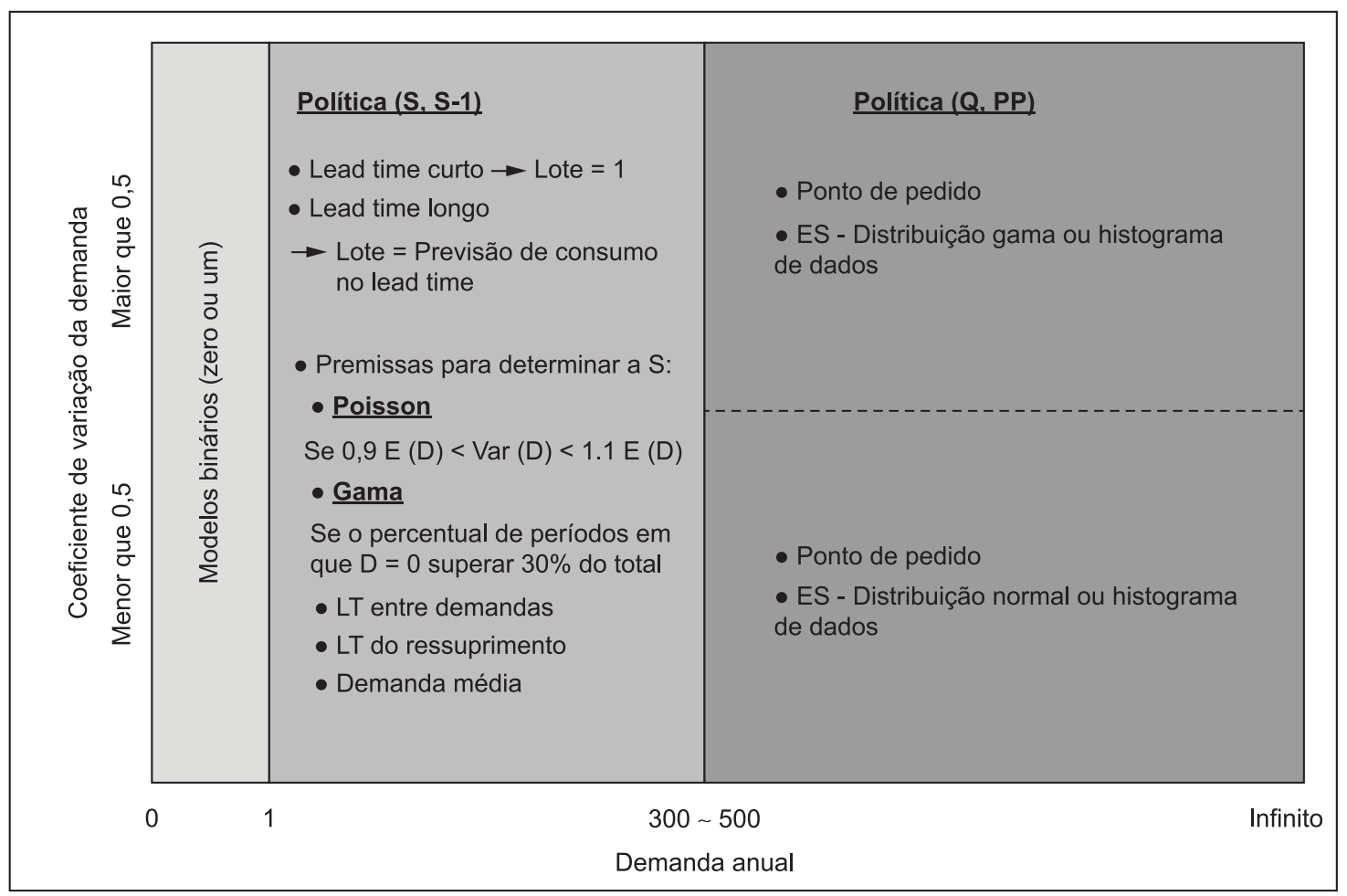

Figura 1. Quadro Conceitual para Gestão de Estoque.

o ponto de corte para o coeficiente de variação da demanda é 0,5.

Mais especificamente, para cada quadrante da demanda anual e do coeficiente de variação da demanda do item, o quadro conceitual contém as funções de distribuição de probabilidade de maior aderência e o modelo de gestão de estoque mais adequado, incorporando todas as considerações teóricas descritas nas seções anteriores.

Não obstante o desenvolvimento de quadros conceituais, Rego et al. (2011) apontam para a necessidade de serem conduzidos estudos enfocando sua aplicabilidade prática nas empresas, com vistas à redução das lacunas entre teoria e prática. Esses estudos de pesquisa-ação permitem ampliar o conhecimento prático na medida em que aspectos envolvendo a compreensão, a complexidade e o esforço gerencial despendido por parte das empresas tornam-se evidentes. Exemplos nesse sentido são encontrados em Cohen et al. (1990), Botter e Fortuin (2000), Strijbosch, Heuts e Schoot (2000), Trimp et al. (2004), Levén e Segerstedt (2004), Wanke (2005), Porras e Dekker (2008), Wagner e Lindemann (2008), Syntetos, Keyes e Babai (2009) e Silva (2009). Nas próximas seções, são apresentados e discutidos os resultados da aplicação prática do quadro conceitual ora proposto numa grande empresa brasileira.

\section{Aplicação prática: descrição de resultados}

Com base no quadro conceitual apresentado na seção 3, foi desenvolvida uma ferramenta em VBA para Excel com a finalidade de auxiliar na segmentação dos itens em estoque a partir de um banco de dados estruturado em planilha eletrônica. Além disso, essa ferramenta também permite responder rapidamente às questões de quanto pedir, quando pedir e quanto manter em estoque de segurança para um número grande de itens, considerando-se um dado nível de serviço que se deseja oferecer aos clientes e à segmentação anteriormente identificada. A ferramenta foi aplicada nos dados reais de uma empresa brasileira, com o objetivo de serem apurados eventuais ganhos oriundos de uma eventual adoção do quadro conceitual para gestão de estoques ora proposto.

A empresa que foi objeto dessa aplicação prática é uma das principais fabricantes mundiais de equipamentos agrícolas e de construção. Ela possui aproximadamente 160 fábricas distribuídas pelo mundo, das quais três estão localizadas no Brasil. Na filial brasileira, trabalham mais de 2.000 funcionários. Além disso, a empresa mantém centralizados nos armazéns da filial brasileira um total de 20.833 diferentes itens. Essa diversidade de itens é considerada pela empresa como fundamental para prover suporte 
às atividades de assistência técnica e aos serviços de pós-venda aos equipamentos por ela comercializados.

Para realizar o comparativo entre a gestão de estoque atualmente verificada na empresa e aquela sugerida pela ferramenta (quadro conceitual), foram utilizados os dados de consumo dos últimos 48 meses para todos os 20.833 itens. Além das informações de consumo, foram utilizados outros dados relevantes para determinação dos modelos, conforme informação da empresa: custo de aquisição do item; custo de ressuprimento/colocação do pedido; custo de indisponibilidade e penalidade; tempo médio de resposta do fornecedor; variância do tempo de resposta do fornecedor; e taxa de oportunidade de manter estoques por ano, para cada um dos diferentes itens.

O primeiro passo na aplicação do quadro conceitual, por intermédio da ferramenta em Excel, consistiu da segmentação dos itens, segundo a classificação de baixíssimo consumo, baixo consumo e consumo de massa, obtendo-se, respectivamente, os seguintes percentuais: $22,0 \% ; 74,5 \%$; e 3,5\%. Além disso, com base nas três categorias de demanda e nas duas de coeficiente de variação, foi analisado o grau de aderência da demanda às distribuições Poisson e Gama, segundo discussão apresentada nas seções 2.2.1 e 2.2.2.

Cabe destacar que, em não se verificando a aderência da demanda à distribuição Poisson ou Gama, supôs-se a demanda aderente à distribuição Normal nos casos em que o coeficiente de variação era inferior a 0,5 .

Todas as análises apresentadas a seguir são relativas aos itens de baixíssimo e baixo consumo, que representam $96,5 \%$ do total de itens, conforme ilustrado na Figura 2.

Com relação aos itens de baixíssimo consumo, observou-se que, para $99,9 \%$ dos itens classificados neste grupo, a empresa deveria manter uma peça em estoque, totalizando 4.586 peças estocadas (uma para cada item). O investimento necessário a ser mobilizado na formação desse estoque é superior a $\mathrm{R} \$ 2.540 .000,00$, caso todas as peças tenham que

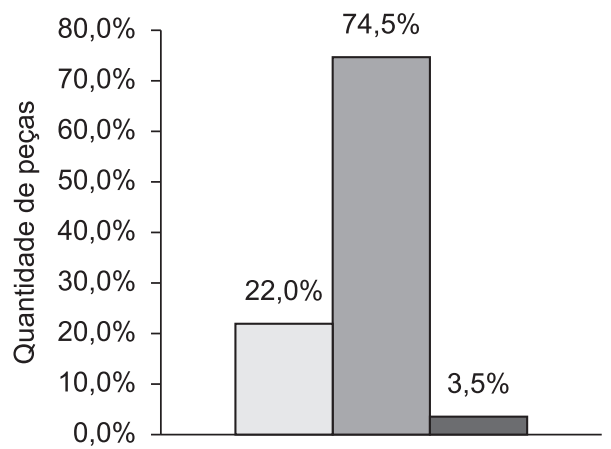

$\square$ Baixíssimo giro $\square$ Baixo giro $\square$ Consumo de massa

Figura 2. Tela de Saída de Resultados - Segmentação e Aderência à Distribuição. ser adquiridas num primeiro momento. Esse valor implica custo de oportunidade anual superior a $\mathrm{R} \$ 563.000,00$.

Já para os itens de baixo consumo aderentes à distribuição Poisson ou Gama, foram calculados os níveis de estoques necessários para atender a três patamares diferentes de níveis de serviço: 90\%, $95 \%$ e $98 \%$ de probabilidade de não faltar o item em estoque. A partir de cada patamar de serviço, foram determinados os custos de oportunidade de manter as peças em estoque, bem como o investimento necessário a ser mobilizado na aquisição desses itens, caso tenham que ser adquiridos simultaneamente num primeiro momento.

Especificamente para o patamar de $90 \%$ de serviço, o investimento necessário a ser mobilizado na formação desse estoque é superior a $\mathrm{R} \$ 84.000 .000,00$, caso todas as peças tenham que ser adquiridas num primeiro momento. Esse valor implica custo de oportunidade anual superior a R \$18.670.000,00. Em média, são mantidas quase 10 peças em estoque para cada item de baixo consumo.

\section{Aplicação prática: discussão dos resultados}

Tendo em vista que a empresa não disponibilizou a real política de estoques adotada, foram assumidas algumas premissas para que fosse possível avaliar se ocorreriam ganhos, tanto em termos financeiros, quanto em termos de nível de serviço, caso fossem adotados os modelos de estoques sugeridos pelo quadro conceitual.

Para os itens de baixíssimo consumo, analisou-se, sob a ótica dos custos totais, a manutenção de nenhuma (CT0) ou de uma (CT1) peça em estoque, conforme as Equações 1 e 3, respectivamente. A manutenção de uma peça em estoque para cada item demonstrou ser a mais adequada, já que apresenta um ganho, dado pelo somatório das diferenças (CT0 - CT1) para todos os itens, igual a $\mathrm{R} \$ 14.429 .517,56$. Os ganhos que podem ser alcançados ao se manter uma peça em estoque para todos os itens se devem principalmente ao fato de que os custos de indisponibilidade e penalidade assumem valores expressivos, tornando-se uma parcela de extrema relevância no cálculo da diferença entre os cenários.

Já no caso dos itens de baixo consumo, que representam $74,5 \%$ do total, considerou-se que a empresa mantinha como estoque-alvo para cada um dos itens o valor médio dos três maiores picos de demanda ocorridos nos últimos 48 meses, prática, por vezes, comum entre as empresas brasileiras (cf. estudo de caso em WANKE, 2011). Dessa forma, para cada item, foi calculado o custo de oportunidade para o nível de estoque sugerido pela ferramenta e o praticado pela empresa. Quando a diferença foi positiva, considerou-se que haveria redução nos custos de oportunidade pela 


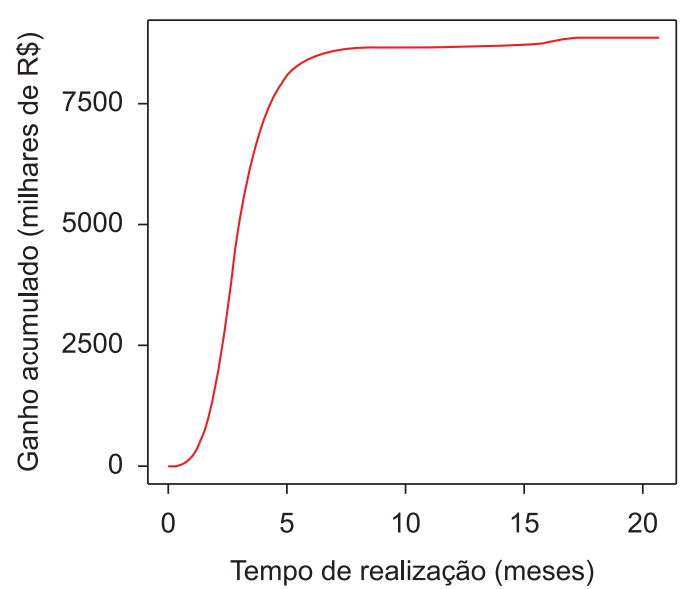

Figura 3. Redução dos Custos de Oportunidade de Manter Estoques no Tempo.

adoção do quadro conceitual. Nesse caso, o montante encontrado foi de quase $\mathrm{R} \$ 8.870 .000,00$, em função de menores níveis de estoque. Similarmente, quando a diferença foi negativa, considerou-se que haveria aumento nos custos de oportunidade em virtude dessa adoção. Nesse caso, o acréscimo total foi de quase $\mathrm{R} \$ 2.230 .000,00$, resultado de maiores níveis de estoque. Cabe salientar que, na maior parte das vezes, os acréscimos verificados nos níveis de estoque decorrem da necessidade de ajuste aos níveis de serviço ao cliente, estipulados, no caso, em $90 \%$.

Com o objetivo de identificar o tempo total para a realização dos ganhos financeiros decorrentes de uma eventual desmobilização de estoques, foi estimado o tempo necessário para que o estoque excedente fosse consumido, tomando como base o consumo médio mensal. A redução de $\mathrm{R} \$ 8.870 .000,00$ nos custos de oportunidade é realizada em, no máximo 20,57 meses, sendo que em seis meses já é possível realizar $\mathrm{R} \$ 8.446 .990,04$, o equivalente a $97,01 \%$ do total, e, em pouco menos de um ano, mais precisamente em 10,29 meses, já é possível realizar 97,89\% dos ganhos, ou seja, R\$ 8.676.001,41 (Figura 3).

O uso da ferramenta, que identifica a aderência das distribuições de itens de baixo giro às distribuições Poisson ou Gama, se mostrou bastante eficiente, uma vez que foram alcançadas economias da ordem de $\mathrm{R} \$ 2.321 .674,55$, a serem realizadas em um prazo máximo de 20,57 meses.

\section{Conclusões}

Uma vasta teoria sobre estoques foi desenvolvida ao longo de cinquenta anos, resultando em inúmeros trabalhos acadêmicos disponíveis na literatura. Contudo, muitos desses resultados teóricos alcançados não se aplicam facilmente na prática das empresas, uma vez que, em sua maioria, assumem premissas que não são verificadas em um mundo corporativo.
Com o intuito de suprir essa lacuna, este trabalho propõe um quadro conceitual para auxiliar na escolha do modelo de gestão de estoque mais adequado.

A partir de diferentes características da demanda, o quadro conceitual proposto evidencia que a premissa da aderência à distribuição Normal nem sempre é válida e que outras distribuições de probabilidade, como Poisson e Gama, devem ser colocadas em perspectiva pelo gestor. Esse trabalho também explorou questões relativas à gestão de estoque de itens de baixo e baixíssimo consumo por meio de uma aplicação prática numa empresa brasileira de equipamentos agrícolas e de construção.

\section{Referências}

BAI, L. Inventory control and demand distribution characterization. School of industrial and Systems Engineering, Georgia Institute of Technology, 2005.

BOTTER, R.; FORTUIN, L. Stocking strategy for service parts - a case study. International Journal of Operations and Production Management, v. 20, n. 6, p. 656-674, 2000. http://dx.doi. org/10.1108/01443570010321612

BALlOU, R. H. Gerenciamento da Cadeia de Suprimentos: Logística Empresarial. 5. ed. Porto Alegre: Bookman, 2006.

BOYLAN, J. E.; SYNTETOS, A. A.; KARAKOSTAS, G. C. Classification for forecasting and stock control: a case study. Journal of the Operational Research Society, v. 59, p. 473-481, 2008. http://dx.doi.org/10.1057/ palgrave.jors. 2602312

BRAGLIA, M.; GRASSI, A.; MONTANARI, R. Multiattribute classification method for spare parts inventory management. Journal of Quality in Maintenance Engineering, v. 10, n. 1, p. 55-65, 2004. http://dx.doi. org/10.1108/13552510410526875

BURGIN, T. A. The gamma distribution and inventory control. Operations Research Quarterly, v. 26, p. 507-525, 1975.

COHEN, M. A. et al. Optimizer: IBM's Multi-Echelon Inventory System for Managing Service Logistics. Interfaces, v. 201, p. 65-82, 1990. http://dx.doi. org/10.1287/inte.20.1.65

COHEN, M. A.; LEE, H. L. Out of Touch with Customer Needs? Spare Parts and After Sales Service. Sloan Management Review, v. 31, n. 2, p. 55-66, 1990.

COHEN, M. A.; ZHENG, Y. S.; AGRAWAL, V. Service parts logistics: a benchmark analysis. IIE Transactions, v. 29, p. 627-639, 1997. http://dx.doi. org/10.1080/07408179708966373

CORRÊA, H. L.; CORRÊA, C. A. Administração de produção e de operações: manufatura e serviços: uma abordagem estratégica. São Paulo: Atlas, 2005.

COSTA, J. C.; GONÇALVES, M. B.; GIACOBO, F. Gestão de estoques de materiais de baixíssimo giro considerando processos críticos para organização. In: SEMINÁRIO DE ADMINISTRAÇÃO, 8., 2005, São Paulo. Anais... São Paulo: USP, 2005.

CROSTON, J. D. Forecasting and Stock Control for Intermittent Demand. Operational Research Quarterly, v. 23, n. 3, p. 289303, 1972. 
DAS, C. Approximate solution to the (Q,r) inventory model for gamma lead time demand. Management Science, v. 22, n. 9, p. 1043-1047, 1976. http://dx.doi. org/10.1287/mnsc.22.9.1043

DEKKER, R.; KLEIJN, M. J.; DE ROOIJ, P. J. A spare parts stocking policy based on equipment criticality. International Journal of Production Economics, v. 5657 , p. $69-77,1998$. http://dx.doi.org/10.1016/ S0925-5273(97)00050-9

DHAKAR, T. S.; SCHMIDT, C. P.; MILLER, D. M. Base stock level determination for high cost low demand critical repairable spares. Computers and Operations Research, v. 21, n. 4, p. 411-420, 1993. http://dx.doi. org/10.1016/0305-0548(94)90028-0

EAVES, A. Forecasting for the ordering and stock-holding of consumable spare parts. 2002. (PhD thesis)-Lancaster University, Department of Management Science, Lancaster, 2002.

EAVES, A. H. C.; KINGSMAN, B. G. Forecasting for the ordering and stock-holding of spare parts. Journal of the Operational Research Society, v. 50, p. 431-437, 2004. http://dx.doi.org/10.1057/palgrave.jors.2601697

EPPEN, G. D.; MARTIN, R. K. Determining safety stock in the presence of stochastic tempo de resposta and demand. Management Science, v. 34, n. 11, p. 1380-1390, 1988. http://dx.doi.org/10.1287/mnsc.34.11.1380

FEENEY, G. J.; SHERBROOKE, C. C. The (s -1, s) inventory policy under compound Poisson demand. Management Science, v. 12, p. 391-411, 1966. http:// dx.doi.org/10.1287/mnsc.12.5.391

GHOBBAR, A. A.; FRIEND, C. H. Evaluation of Forecasting Methods for Intermittent Parts Demand in the Field of Aviation: a Predictive Model. Computers \& Operations Research, v. 30, p. 2097-2114, 2003. http://dx.doi. org/10.1016/S0305-0548(02)00125-9

GOMES, A. V. P.; WANKE, P. Modelagem da gestão de estoques de peças de reposição através de cadeias de Markov. Gestão \& Produção, v. 15-1, p. 57-72, 2008.

GOMEZ, G. C. G. Lumpy demand characterization and forecasting performance using self-adaptive forecasting models and Kalman Filter. 2008. $121 \mathrm{f}$. Dissertation (Master in Industrial Engineering)-The University of Texas, El Paso, 2008.

GUTIERREZ, R. S.; SOLIS, A. O.; MUKHOPADHYAY, S. Lumpy demand forecasting using neural networks. International Journal of Production Economics, v. 111, p. 409-420, 2008. http://dx.doi.org/10.1016/j. ijpe.2007.01.007

HADLEY, G.; WHITIN, M. A family of inventory models. Management Science, v. 7, n. 4, p. 351-371, 1961. http://dx.doi.org/10.1287/mnsc.7.4.351

HARRIS, F. W. How many parts to make at once. Factory, The Magazine of Management, v. 10, n. 2, p. $135-136,1913$

HAX, A.; CANDEA, D. Production and Inventory Management. Prentice-Hall, Inc., 1984.

HILL, R. M.; OMAR, M.; SMITH, D. K. Stock replenishment policies for a stochastic exponentially-declining demand process. European Journal of Operational Research, v. 116, p. 374-388, 1999. http://dx.doi.org/10.1016/ S0377-2217(98)00223-9

HOPP, W. J.; SPEARMAN, M. L. Factory Phisics. 3rd ed. New York: McGraw-Hill, 2008. 720 p.
HUA, Z. S. et al. A new approach of forecasting intermittent demand for spare parts inventories in the process industries. Journal of the Operational Research Society, v. 58, p. 52-61, 2007. http://dx.doi.org/10.1057/ palgrave.jors. 2602119

HUISKONEN, J. Maintenance spare parts logistics: Special characteristics and strategic choices. International Journal of Production Economics, v. 71, p. 125-133, 2001 http://dx.doi.org/10.1016/ S0925-5273(00)00112-2

JANSSEN, F.; HEUTS, R.; KOK, A. On the (R, s, Q) inventory model when demand is modelled as a compound Bernoulli process. European Journal of Operational Research, v. 104, p. 423-436, 1998. http:// dx.doi.org/10.1016/S0377-2217(97)00009-X

KEATON, M. Using the Gamma distribution to model demand when tempo de resposta is random. Journal of Business Logistics, v. 16, n. 1, p. 107-131, 1995.

KRUPP, J. Safety stock management. Production and Inventory Management Journal, v. 38, n. 3, p. 11-18, 1997.

KUMAR, S. Parts Management Models and Applications. New York: Springer, 2005. 222 p.

LEVINE, D. M. et al. Estatística - Teoria e Aplicações. 3. ed. Rio de Janeiro: LTC, 2005.

LAU, H.-S. Toward a Stock Control System under Non-Normal Demand and Lead-time Uncertainty. Journal of Business Logistics, v. 10, n. 1, p. 88-103, 1989.

LEVÉN, E.; SEGERSTEDT, A. Inventory control with a modified Croston procedure and Erlang distribution. International Journal of Production Economics, v. 90, p. 361-367, 2004. http://dx.doi.org/10.1016/ S0925-5273(03)00053-7

LOVE, S. Inventory Control. New York: McGrawHill, 1979. 273 p.

MENTZER, J. T.; KRISHNAN, R. The effect of the assumption of normality on inventory control/customer Service. Journal of Business Logistics, v. 6, n. 1, p. 101-120, 1988.

MOORS, J. J. A.; STRIJBOSCH, L. W. G. Exact fill rates for $(\mathrm{R} ; \mathrm{s} ; \mathrm{S})$ inventory control with Gama distributed demand. Journal of the Operational Research Society, v. 53, n. 11, p. 1268-1274, 2002. http://dx.doi.org/10.1057/ palgrave.jors. 2601441

MUCKSTADT, J. A. Analysis and Algorithms for Service Parts Supply Chains. New York: Springer, 2004. 277 p.

NAMIT, K.; CHEN, J. Solutions to the inventory model for gamma tempo de resposta demand. International Journal of Physical Distribution \& Logistics Management, v. 29, n. 2, p. 138-154, 1999. http:// dx.doi.org/10.1108/09600039910264713

PORRAS, E.; DEKKER, R. An inventory control system for spare parts at a refinery: An empirical comparison of different re-order point methods. European Journal of Operational Research, v. 184, n. 1, p. 101-132, 2008. http://dx.doi.org/10.1016/j.ejor.2006.11.008

REGATTIERI, A. et al. Managing lumpy demand for aircraft spare parts. Journal of Air Transportation Management, v. 11, p. 426-431, 2005. http://dx.doi. org/10.1016/j.jairtraman.2005.06.003

REGO, J. R. A lacuna entre a Teoria de Gestão de Estoques e a prática empresarial na reposição de peças em concessionárias de automóveis. 2006. 115 f. Dissertação (Mestrado em Administração)-Faculdade de 
Economia, Administração e Contabilidade, Universidade de São Paulo, São Paulo, 2006.

REGO, J. R.; MESQUITA, M. A. Controle de estoque de peças de reposição em local único: uma revisão da literatura. Produção, v. 21, n. 4, p. 645-666, 2011. http://dx.doi.org/10.1590/S0103-65132011005000002

ROSA, H.; MAYERLE, S. F.; GONÇALVES, M. B. Controle de estoque por revisão contínua e revisão periódica: uma análise comparativa utilizando simulação. Produção, v. 20, n. 4, dez. 2010. http://dx.doi.org/10.1590/ S0103-65132010005000052

SHERBROOKE, C. C. Optimal Inventory Modeling of Systems - Multi-Echelon Techniques. 2nd ed. Boston: Kluwer, 2004. 332 p. PMid:15277561.

SILVA, G. L. C. Modelo de estoque para peças de reposição sujeitas à demanda intermitente e leadtime estocástico. 2009. 75 f. Dissertação (Mestrado em Engenharia de Produção)-Escola de Engenharia, Universidade Federal de Minas Gerais, 2009.

SILVER, E.; HO, C.-M.; DEEMER, R. Cost minimizing inventory control of items having a special type of erratic demand pattern. INFOR, v. 9, p. 198-219, 1971.

SILVER, E. A.; PETERSON, R. Decision Systems for Inventory Management and Production Planning. 2nd ed. New York: John Wiley \& Sons, 1985.

SILVER, E. A.; PETERSON, R.; PYKE, D. F. Inventory Management and Production Planning and Scheduling. 3rd ed. New York: John Wiley \& Sons, 1998. $754 \mathrm{p}$.

SPEH, T. W.; WAGENHEIM, G. D. Demand and lead-time uncertainty: The impacts on physical distribution performance and management. Journal of Business Logistics, v. 1, n. 1, p. 95-113, 1978.

STRIJBOSCH, L. W. G.; HEUTS, R. M. J.; SCHOOT, E. H. M. A combined forecast-inventory control procedure for spare parts. Journal of Operational Research Society, v. 51, p. 1184-1192, 2000

SYNTETOS, A. A.; BOYLAN, J. E. On the bias of intermittent demand estimates. International Journal of Production Economics, v. 71, n. 1-3, p. 457-466, 2001. http://dx.doi.org/10.1016/S0925-5273(00)00143-2

SYNTETOS, A. A.; BOYLAN, J. E.; CROSTON, J. D. On the categorization of demand patterns. Journal of Operational Research Society, v. 56, p. 495-503, 2005. http://dx.doi.org/10.1057/palgrave.jors.2601841

SYNTETOS, A. A.; KEYES, M.; BABAI, M. Z. Demand categorization in a European spare parts logistics network. International Journal of Operations \& Production Management, v. 29-3, p. 292-316, 2009.

TAVARES, L. V.; ALMEIDA, L. T. A binary decision model for stock control os very slow moving items. Operational Research Society, v. 34, n. 3, p. 249-252, 1983.

TEUNTER, R. H.; DUNCAN, L. Forecasting intermittent demand: a comparative study. Journal of the Operational Research Society, v. 60-3, p. 321-329, 2009. http:// dx.doi.org/10.1057/palgrave.jors.2602569

TRIMP, M. E. et al. Optimise initial spare parts inventories: an analysis and improvement of an electronic decision tool. Erasmus Universiteit Rotterdam, 2004.
Report Econometric Institute EI:2004-52. Disponível em: $<$ http://publishing.eur.nl/ir/repub/asset/1830/ei200452. pdf.> Acesso em: 08 ago. 2009.

TYWORTH, J. E. Modeling transportation-inventory tradeoffs in a stochastic setting. Journal of Business Logistics, v. 13, n. 2, p. 97-124, 1992.

TYWORTH, J. E.; O'NEILL L. Robustness of the normal approximation of lead-time demand in a distribution setting. Naval Research Logistics, v. 44, n. 6, p. 165-86, 1997. http://dx.doi.org/10.1002/(SICI)15 20-6750(199703)44:2<165::AID-NAV2>3.0.CO;2-7

TYWORTH, J. E.; GANESHAN, R. A note on solutions to the $<\mathrm{Q}, \mathrm{R}>$ inventory model for gamma lead-time demand. International Journal of Physical Distribution \& Logistics Management, v. 30, n. 6, p. 534-539, 2000. http://dx.doi.org/10.1108/09600030010340897

YEH, Q.-J. A practical implementation of gamma distribution to the reordering decision of an inventory control problem. Production and Inventory Management Journal, v. 37, n. 8, p. 51-57, 1997.

YEH, Q.-J.; CHANG, T. P.; CHANG, H. C. An inventory control model with gamma distribution. Microelectronics and Reliability, v. 37, n. 8, p. 1197-1201, 1997. http:// dx.doi.org/10.1016/S0026-2714(96)00295-8

WAGNER, S. M.; LINDEMANN, E. A case study-based analysis of spare parts management in the engineering industry. Production Planning \& Control, v. 19-4, p. 397-407, 2008.

WALKER, J. Base stock level determination for "insurance type" spares. International Journal of Quality \& Reliability Management, v. 14, n. 6, p. 569-574, 1997. http://dx.doi.org/10.1108/02656719710186182

WANKE, P. Metodologia para gestão de estoques de peças de reposição: um estudo de caso em empresa brasileira. Revista Tecnologística, p. 60-65, dez. 2005.

WANKE, P. The impact of different demand allocation rules on total stock levels. Pesquisa Operacional, v. 30, n. 1, p. 33-52, 2010.

WANKE, P. Gestão de estoques na cadeia de suprimentos: decisões e modelos quantitativos. 3. ed. São Paulo: Atlas, 2011.367 p. (Coleção COPPEAD de Administração).

WANKE, P. Product, operation, and demand relationships between manufacturers and retailers. Transportation Research Part E: Logistics and Transportation Review, v. 48, n. 1, p. 340-354, 2012. http://dx.doi.org/10.1016/j. tre.2011.07.010

WANKE, P. F. \& SALIBY, E. Consolidation effects: Whether and how inventories should be pooled. Transportation Research Part E: Logistics and Transportation Review, v. 45, n. 5, p. 678-692, 2009. http://dx.doi.org/10.1016/j. tre.2009.01.006

WILLEMAIN, T. R.; SMART, C. N.; SCHWARZ, H. F. A new approach to forecasting intermittent demand for service parts inventories. International Journal of Forecasting, v. 20, p. 375-387, 2004 http://dx.doi. org/10.1016/S0169-2070(03)00013-X

WILLIAMS, T. M. Stock Control with Sporadic and Slow-Moving Demand. The Journal of the Operational Research Society, v. 35-10, p. 939-948, 1984. 
